Research Paper

\title{
Targeting LncRNA-MALAT1 suppresses the progression of osteosarcoma by altering the expression and localization of $\beta$-catenin
}

\author{
Zhi-Chang Zhang ${ }^{1 *}$, Chun Tang ${ }^{2 *}$, Yang Dong${ }^{1}$, Jing Zhang1, Ting Yuan ${ }^{1 凶}$, Xiao-Lin Li $^{1 凶}$ \\ 1. Department of Orthopaedic Surgery, Shanghai Jiao Tong University Affiliated Sixth People's Hospital, 600 Yishan Road, Shanghai 200233, China; \\ 2. Department of Nursing, Guangming Traditional Chinese Medicine Hospital, Pudong New Area, Shanghai 201300, China. \\ ${ }^{*}$ Co-first authors: These authors contributed equally to this work. \\ $\square$ Corresponding authors: Xiao-Lin Li, Department of Orthopaedic Surgery, Shanghai Jiao Tong University Affiliated Sixth People's Hospital, 600 Yishan Road, \\ Shanghai 200233, China E-mail: lixiaolin@sjtu.edu.cn Ting Yuan, Department of Orthopaedic Surgery, Shanghai Jiao Tong University Affiliated Sixth People's \\ Hospital, 600 Yishan Road, Shanghai 200233, China E-mail: terrenceyuan@gmail.com \\ (C) Ivyspring International Publisher. This is an open access article distributed under the terms of the Creative Commons Attribution (CC BY-NC) license \\ (https://creativecommons.org/licenses/by-nc/4.0/). See http://ivyspring.com/terms for full terms and conditions.
}

Received: 2017.07.27; Accepted: 2017.10.24; Published: 2018.01.01

\begin{abstract}
Osteosarcoma (OS), which affects adolescents especially during a growth spurt, has the highest incidence of any primary malignant bone tumour, and a high rate of early metastasis leading to a very poor prognosis. In recent years, non-coding RNAs, especially long non-coding RNAs (IncRNAs) have attracted more and more attention as novel epigenetic regulators in a variety of tumours, including OS. Most recently, metastasis-associated lung adenocarcinoma transcript 1 (MALATI) was found to play an important role in OS progression by modulating the enhancers of zeste homolog 2 (EZH2). Furthermore, MALAT1 could inhibit the expression of E-cadherin and promote the expression of $\beta$-catenin, and this phenomenon might be the outcome of MALATl-induced EZH2 activation. In this study, we investigated the vital function of MALAT1 in the progression of OS and its potential leading mechanism, altering the expression and localization of $\beta$-catenin via epigenetic transcriptional regulation by interacting with EZH2. With the help of MALAT1 silencing using small interfering RNAs (siRNAs), the loss of E-cadherin of MNNG/HOS cells was rescued, and the abnormal expression and localization of $\beta$-catenin were corrected at the same time. Overall, our research showed promising potential for new treatment strategies based on epigenetic regulation targeting MALAT1, which will not only coordinate with the patient's immune system, but also eliminate OS in conjunction with chemotherapy.
\end{abstract}

Key words: osteosarcoma, long non-coding RNA, MALAT1, E-cadherin, $\beta$-catenin.

\section{Introduction}

Osteosarcoma (OS), also known as osteogenic sarcoma, usually arises during an adolescent growth spurt, and has the highest incidence of primary malignant bone tumours worldwide [1-3]. The five-year survival rate of patients with non-metastatic disease is around $60-70 \%$; however, prognosis is significantly worse in patients with metastases. $[4,5]$ Unfortunately, in approximately $80 \%$ of OS patients, malignant tumour cells have spread to other organs at the time of diagnosis. [6] Thus, future OS treatment depends on therapeutic strategies based on a thorough understanding of the disease. Following increasing attention to the molecular mechanism of OS progression, a wide range of current research is attempting to establish the potential biological pathways related to new therapeutic approaches. [7]

Originally, the accepted central dogma was that after DNA is transcribed into messenger RNA (mRNA), gene information is then ultimately translated into proteins which control all cellular functions. However, a growing amount of recent research has indicated that non-coding RNAs play 
important roles in both normal physiological processes and disease progression, including malignant tumours. [8] Long non-coding RNAs (lncRNAs) are defined as non-coding transcripts, which are expressed in differentiated tissues or specific malignant tumour types, have no protein-coding potential, and comprise more than 200 nucleotides. [9-11] Recent studies indicate that lncRNAs participate in malignant tumour genesis and metastasis, including of OS. [12, 13]

Originally, metastasis-associated lung adenocarcinoma transcript 1 (MALAT1), part of the lncRNA family, was found to be a reliable lncRNA biomarker of lung cancer. [14] Subsequently, increasing evidence demonstrated a positive correlation between the expression level of MALAT1 and the disease progression of OS. [15] It was therefore concluded that MALAT1 could enhance OS cell proliferation and migration. [16-18] However, opinions still vary greatly regarding the detailed mechanism of the involvement of MALAT1 in OS progression, and more in-depth research is needed.

In colorectal cancer patients, research indicates that MALAT1 might interact with the enhancer of zeste homolog 2 (EZH2) and participate in potential transcriptional regulation. [19] Furthermore, research demonstrates that MALAT1 can inhibit the expression levels of E-cadherin and promote the expression levels of $\beta$-catenin. [20-22] In fact, E-cadherin negatively regulates $\beta$-catenin signalling by forming a $\beta$-catenin/E-cadherin complex at the cell surface; and down-regulation of E-cadherin leads to enhanced $\beta$-catenin signalling via disruption of formation of the $\beta$-catenin/E-cadherin complex. [23, 24] Hence, we assume that MALAT1 is involved in the progression of OS by dramatically enhancing the $\beta$-catenin signalling pathway. The latter specifically involves simultaneous up-regulation of the expression of $\beta$-catenin and disruption of the re-location of $\beta$-catenin onto the cell membrane.

In the present study, we used a modified small interfering RNA (siRNA), with enhanced stability and potency in vivo. [25] This was used to inhibit OS progression by blocking the aberrant activation of $\beta$-catenin signalling, and to explore its therapeutic mechanism, in order to exploit a new therapeutic strategy to cure OS.

\section{Materials and Methods}

\section{Cell Culture}

MNNG/HOS, one of the most widely-acknowledged human OS cell lines [26, 27], was kindly provided by the Cell Bank (Chinese Academy of Sciences, Shanghai, China) and used in this study. MNNG/HOS cell lines were cultured in DMEM (Thermo Fisher Scientific, Waltham, MA, USA) supplemented with $10 \%$ foetal bovine serum (FBS; Thermo Fisher Scientific) in a humidified $37^{\circ} \mathrm{C}$ incubator with $5 \% \mathrm{CO}_{2}$.

\section{RNAi}

Ambion ${ }^{\circledR}$ In Vivo Pre-designed siRNA for MALAT1 (siMALAT1; siRNA ID: n511399) and Ambion ${ }^{\circledR}$ In Vivo Negative Control siRNA (siCtrl) were purchased from Thermo Fisher Scientific. For in vitro transfection the siRNA (final concentration: 50 $\mathrm{nM}$ ) was combined with Lipofectamine ${ }^{\mathrm{TM}} 2000$ Transfection Reagent (Invitrogen, Thermo Fisher Scientific) following the manufacturer's instructions. For in vivo transfection the siRNA was combined with Invivofectamine ${ }^{\mathrm{TM}} \quad 3.0$ Reagent (Invitrogen) in accordance with the manufacturer's instructions.

\section{Inhibitor}

GSK343, a highly-specific inhibitor of EZH2, [28, 29] was purchased from Selleckchem (Houston, TX, USA). The concentration used in this study was 10 $\mu \mathrm{M}$. The disposition and usage were in accordance with the recommendations made in the appendant handbook.

\section{RNA isolation and reverse transcription (RT)-polymerase chain reaction (PCR)}

Total RNA extraction with TRIzol Reagent (Invitrogen), followed by cDNA synthesis using a TransScript All-in-One First-Strand cDNA Synthesis SuperMix (Transgen Biotech, Beijing, China), was performed in accordance with the manufacturer's guidebook and as described previously. [30] PCR was performed using a Bio-Rad PCR instrument (Bio-Rad, Hercules, CA, USA) with 2×Taq PCR Master Mix (Solarbio, Beijing, China) following the manufacturer's instructions. below:

The primers for the PCR procedure are described

MALAT1-FOR: 5'-GCA TTT TGG GAT GGT CTT AA-3'

MALAT1-REV: 5'-CAG CGG TAC ACT CCT TCT CT-3'

GAPDH-FOR: 5'-GGG AAG GTG AAG GTC GGA GT-3

GAPDH-REV: 5'-GGG GTC ATT GAT GGC AAC A-3'

\section{Polyacrylamide gel electrophoresis (PAGE)}

PAGE analysis of the PCR products was performed using a pre-formed PAGE gel (Solarbio) following the instructions provided in the handbook. The sample preparation procedure was performed using 10,000× SYBR Green I, sterilized deionized 
water and $6 \times$ DNA Loading Buffer (all from Solarbio) following the manufacturer's instructions. After electrophoresis at $150 \mathrm{~V}$ for 40 minutes, the gel was photographed using a Gel Doc ${ }^{\mathrm{TM}} \mathrm{XR}+$ system (Bio-Rad).

\section{Cell Counting Kit-8 (CCK-8) assay}

Cell counting kit-8 was purchased from Dojindo Molecular Technologies (Kumamoto, Japan). After transfection with siRNA or siCtrl for 24 hours, a total of $5 \times 10^{3}$ cells per well were seeded into a 96-well plate and cultured with or without GSK343. A blank group served as control without cells. On days zero, one, two and three, $10 \mu \mathrm{L}$ CCK-8 solution was added to each well, and the absorbance at $450 \mathrm{~nm}$ was measured using a microplate reader after one hour of incubation at $37^{\circ} \mathrm{C}$. Throughout the process, the inhibitor group was cultured in medium containing the inhibitor. The difference in optical density value (OD) between the sample group and the blank group, which is proportional to the living cell number, was calculated.

\section{Migration assay}

After transfection with siRNA or siCtrl for 48 hours with or without GSK343, a total of $5 \times 10^{5}$ cells per well were seeded onto the upper surface of the upper chamber of $8 \mu \mathrm{m}$ transwell plates (Corning, Corning, NY, USA). The individual steps were performed as described in a previous study. [30] After incubation for 6 hours, the insert was stained with $0.5 \%$ crystal violet before the stained cells were observed under an optical microscope.

\section{Annexin-V/propidium iodide (PI) assay using flow cytometry (FCM)}

For the study of the apoptotic process, flow cytometry with Annexin-V and PI double staining is a well-received method. [31, 32] An annexin V-FITC early apoptosis detection kit (Cell Signalling Technology, Danvers, MA, USA) was used in this study, in accordance with the manufacturer's guidebook.

\section{Immunoblotting and antibodies}

The separate extraction of membrane proteins, cytoplasmic proteins and nuclear proteins was performed using the Subcellular Protein Fractionation Kit for Cultured Cells (Thermo-Fisher Scientific) according to the detailed instructions provided by the manufacturer. The detailed procedures of western blotting were as described previously [33]. The primary antibodies used in this study, including E-Cadherin, $\beta$-Catenin, $\mathrm{Na} / \mathrm{K}$-ATPase, GAPDH, Histone $\mathrm{H} 3$ and $\mathrm{c}-\mathrm{Myc}$, were purchased from Cell Signalling Technology (CST; Boston, MA, USA).

\section{Nude mouse transplantation tumour assay}

The nude mouse transplantation tumour assay is a well-recognized model for observing the outcome of oncotherapy. [27, 34] Male nude mice, aged approximately 6 weeks, were kept in an SPF-grade pathogen-free animal laboratory with laminar flow equipment and 12 hour light-dark cycles. All experimental and animal care procedures were approved by the Animal Research Ethics Committee of Shanghai Sixth People's Hospital and performed in accordance with the Interdisciplinary Principles and Guidelines for the Use of Animals in Research, Testing, and Education by the New York Academy of Sciences, ARRIVE (Animal Research: Reporting of In Vivo Experiments) Guidelines, and the National Institutes of Health guide for the care and use of Laboratory animals.

Heterotransplantation of MNNG/HOS was performed by subcutaneous injection of $5 \times 10^{6}$ MNNG/HOS cells around the left side of the neck. Seven days after injection, when OS tumours had reached approximately $3 \mathrm{~mm}^{3}$, mice were randomly divided into two groups ( $\mathrm{n}=5$ per group). On days 7 , 12 and 17 after inoculation, intra-tumour injections were performed with siCtrl $(10 \mathrm{mg} / \mathrm{kg})$ or siMALAT1 $(10 \mathrm{mg} / \mathrm{kg})$ after siRNAs were mixed with Invivofectamine ${ }^{\mathrm{TM}}$ and diluted using normal saline.

Twenty-one days after inoculation, the mice were sacrificed before the tumours were carefully dissected. The samples were embedded in paraffin and sectioned as described previously. [30]

\section{Histological staining and immunofluorescence staining in paraffin sections}

The detailed procedures of hematoxylin-eosin (HE) staining and immunofluorescence staining were as described previously [30,33].

The primary antibodies used for the immunostaining of paraffin sections were Ki67 (Abcam, Cambridge, MA, USA), cleaved-caspase-3 (CST) and $\beta$-catenin (CST).

\section{Statistical analysis}

Data are described as means \pm standard deviation (SD). The statistical significance of differences between means was evaluated by one-way analysis of variance (ANOVA). Statistical analysis was performed using RStudio (Boston, MA, USA). Statistical significance was defined as $P$ values $<0.05$.

\section{Results}

The MALAT1/EZH2 axis participates in OS cell proliferation, migration, and cell survival in vitro

The expression level of MALAT1 was measured 
by PAGE using the products of RT-PCR. We confirmed that the expression level of MALAT1 was dramatically higher in MNNG/HOS, a human osteosarcoma cell line, than in human bone marrow stem cells (BMSCs), normal bone marrow cells with osteogenic potential (Fig. 1A).

In preparation for the subsequent experiments, the efficiency of siMALAT1 was confirmed and it was demonstrated that siRNA suppressed the expression of MALAT1 with satisfactory efficiency (Fig. 1B).

To confirm that the MALAT1/EZH2 axis is essential in the progression of OS, we used siMALAT1 and GSK343, a highly-specific inhibitor of EZH2, $[28,29]$ to block the MALAT1/EZH2 axis and observe the influence on activity representative of malignant tumour progression, including proliferation and migration, using CCK-8 assay and Transwell ${ }^{\circledR}$ assay. We found that blocking the MALAT1/EZH2 axis dramatically inhibited both the proliferation (Fig. 1C) and migration (Fig. 1D) of MNNG/HOS cells, signifying that MALAT1 is a potential target for the gene therapy of OS.
A

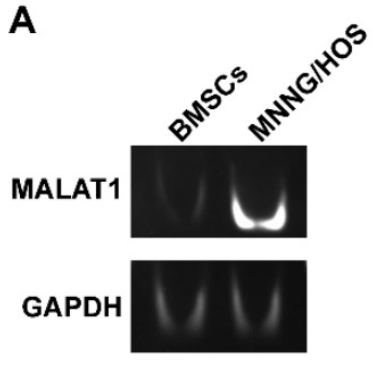

B

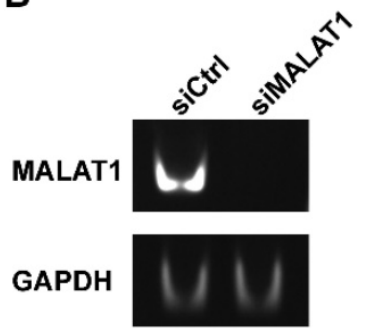

C

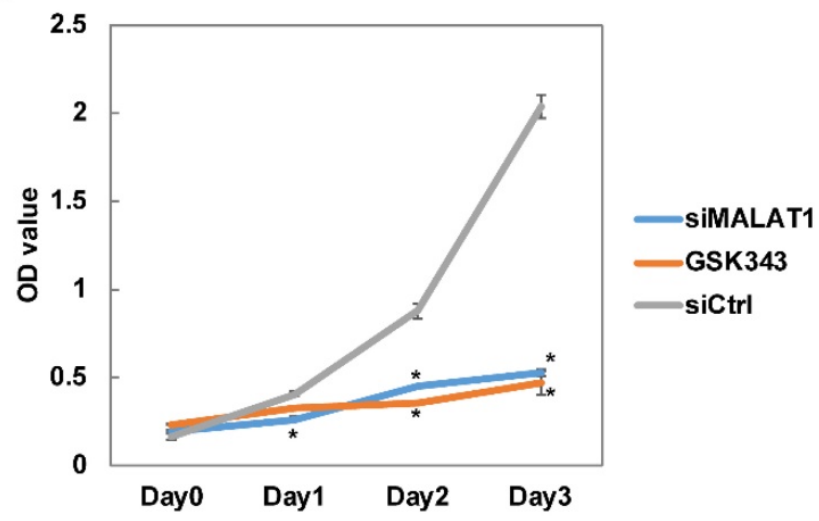

D

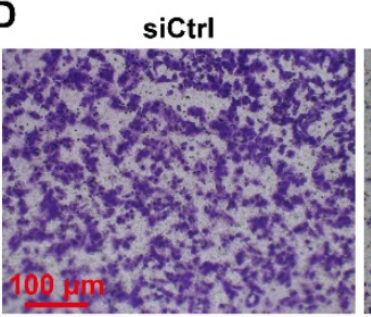

SIMALAT1

GSK343
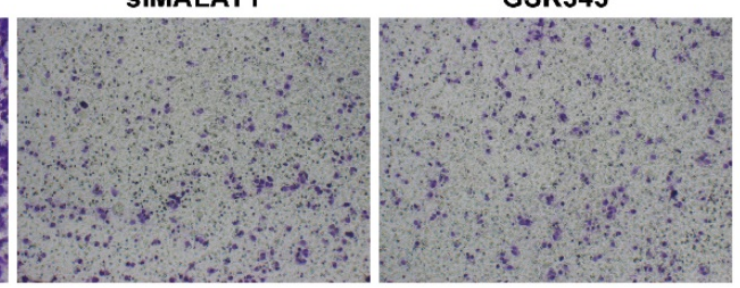

E

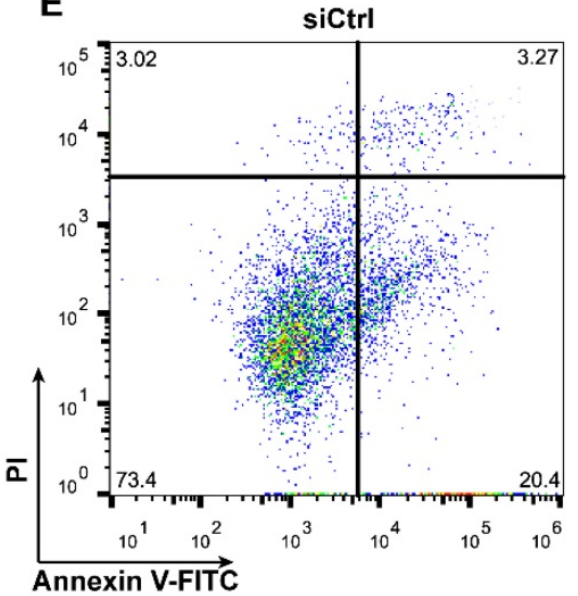

SIMALAT1

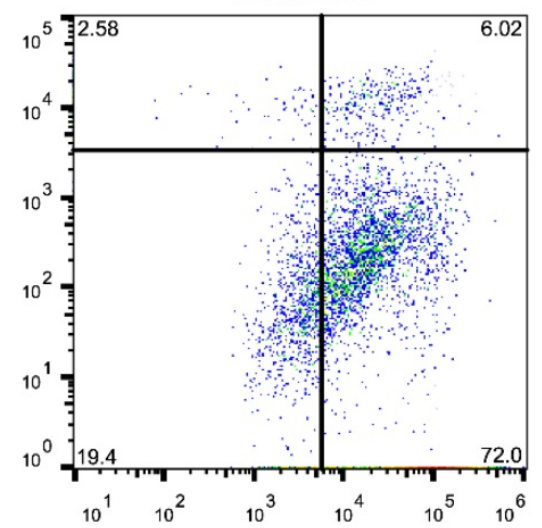

GSK343

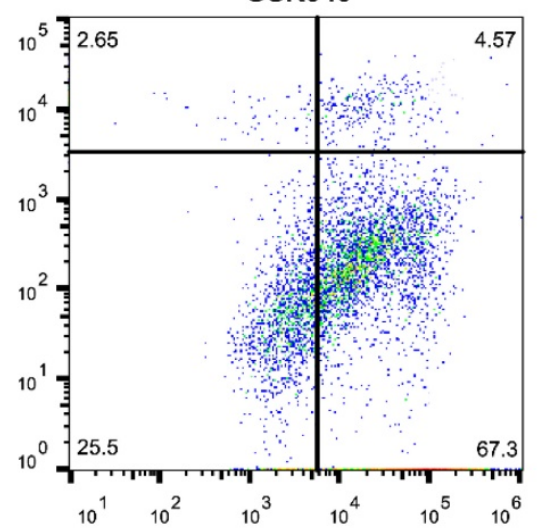

Figure 1. The MALAT1/EZH2 axis participates in OS cell proliferation, migration, and cell survival in vitro. (A) The expression level of MALAT1 in MNNG/HOS and BMSCs detected by PAGE. (B) The efficiency of siMALATI detected by PAGE. (C) The proliferative ability measured by CCK-8 assay. $* P<0.05$ compared to siCtrl. (D) Migration ability measured by Transwell $\circledast$ assay. (E) The apoptotic rate of MNNG/HOS cells after different treatments following $48 \mathrm{~h}$ serum starvation, measured by flow cytometry with Annexin-V/PI staining. 
Since the tumour microenvironment has an extremely low supply of serum components caused by the uncontrolled proliferation of malignant tumours, serum starvation can be used to simulate this barren microenvironment and thus observe the special mechanisms involved in the anti-apoptotic action of malignant tumours. [35] After 48 hours of serum starvation, a large proportion of MNNG/HOS cells were able to tolerate this barren simulated tumour microenvironment, while blocking the MALAT1/EZH2 axis could lead to maladjustment (Fig. 1E).

\section{The expression and re-localization of $\beta$-catenin are regulated by the MALAT1/EZH2 axis in vitro}

When considering the triangular relations among EZH2, E-cadherin and $\beta$-catenin, we assumed that MALAT1 could promote the expression level of $\beta$-catenin and, beyond that, increase the effectiveness of $\beta$-catenin by inhibiting the expression level of E-cadherin and consequently decreasing the proportion of E-cadherin-binding $\beta$-catenin.

After blocking the MALAT1/EZH2 axis, the expression level of $\beta$-catenin and c-Myc, a downstream mediator of $\beta$-catenin, was significantly decreased, as expected. Furthermore, the expression level of E-cadherin, which was once suppressed by the MALAT1/EZH2 axis, increased noticeably (Fig. 2A).

The membrane proteins, cytoplasmic proteins and nuclear proteins were extracted for analysis. GAPDH was used as a sign of contamination by cytoplasmic proteins. $\mathrm{Na} / \mathrm{K}$-ATPase was used as a specific marker of surface protein, while histone $\mathrm{H} 3$ was used as a specific marker of nuclear protein. It is significant, and very encouraging, that $\beta$-catenin was translocated onto the membrane along with the increase of E-cadherin (Fig. 2B).

\section{siMALAT1 prevents OS progression by participating in OS cell proliferation, migration and cell survival in vivo}

Our results proved that siMALAT1 could achieve the desired effects in vivo. The tumour size was significantly smaller in the siMALAT1 group than in the control group (Fig. 3A).

The in vivo proliferation rate of OS cells was evaluated after Ki67 immunofluorescence staining and results showed that siMALAT1 dramatically decreased the proliferation rate of OS cells in vivo, consistent with the in vitro results (Fig. 3B).

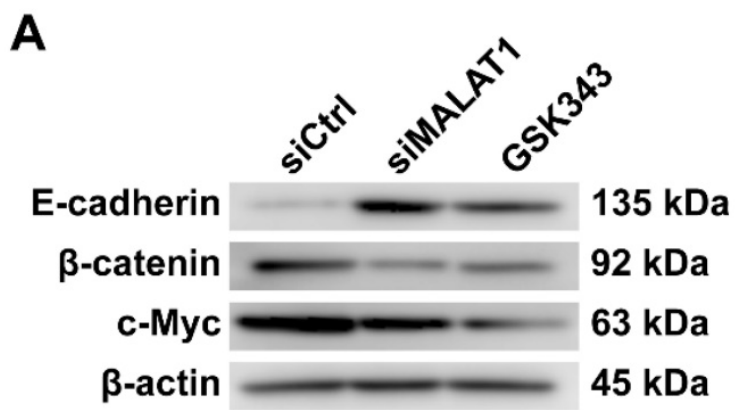

B

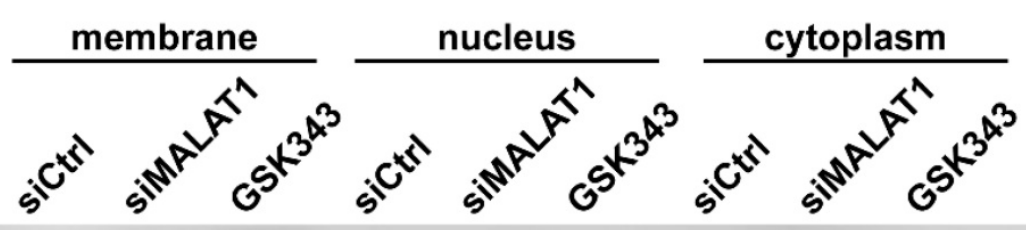

E-cadherin

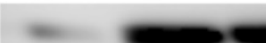

s

$135 \mathrm{kDa}$

$\beta$-catenin

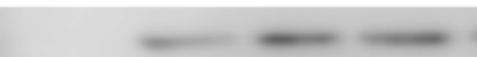

$92 \mathrm{kDa}$

$\mathrm{Na} / \mathrm{K}$-ATPase

$100 \mathrm{kDa}$

Histone H3

$17 \mathrm{kDa}$

GAPDH

37 kDa

Figure 2. The expression and re-localization of $\beta$-catenin are regulated by the MALAT1/EZH2 axis in vitro. (A) Total protein level of MNNG/HOS cells after different treatments, measured by western blotting. (B) Separate extracts of membrane proteins, cytoplasmic proteins and nuclear proteins analysed by western blotting. 
A

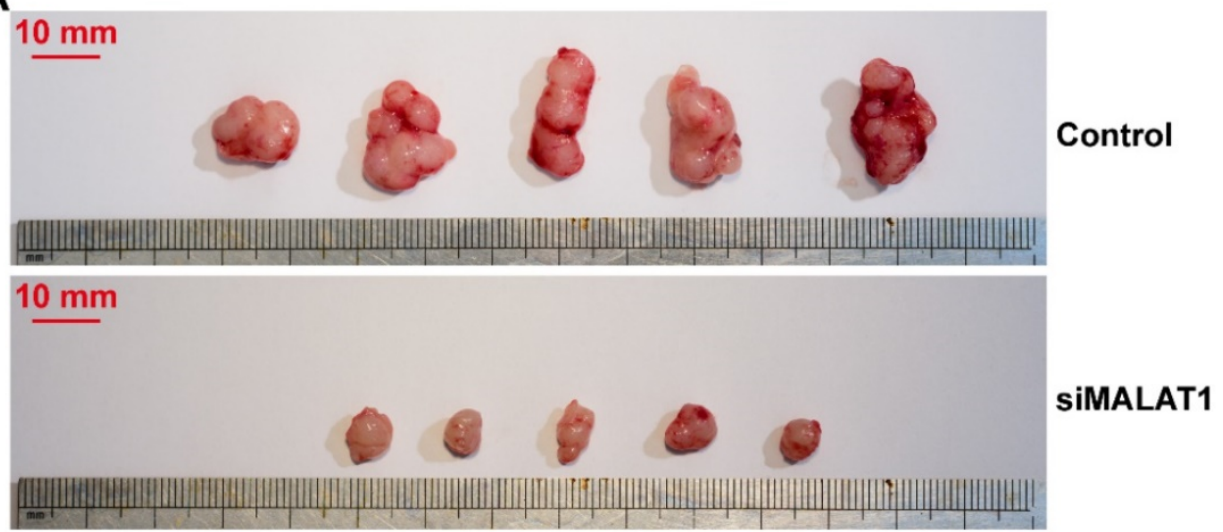

B

Control

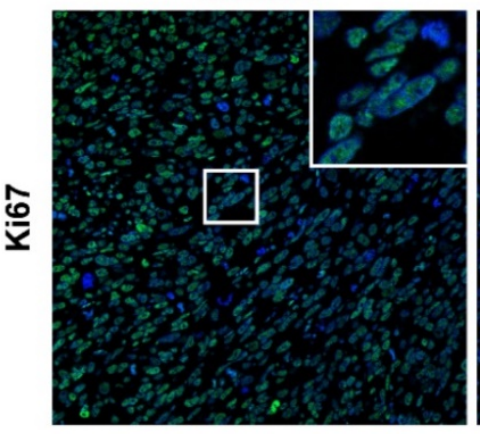

SIMALAT1

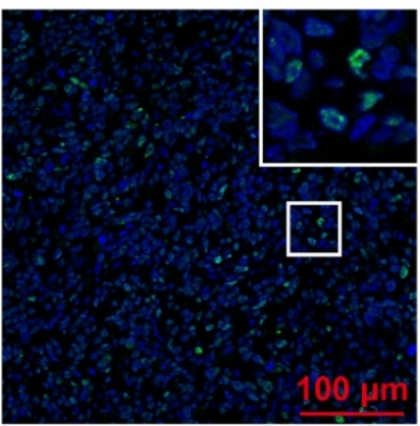

C

Control

SIMALAT1
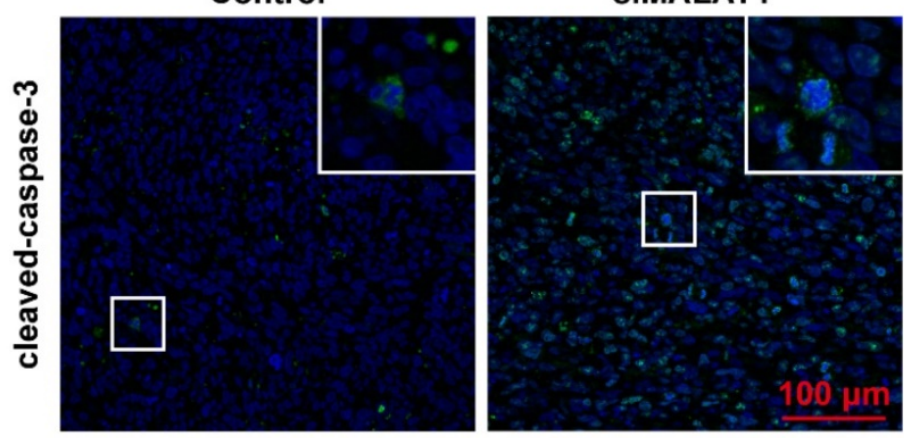

D

Control

SIMALAT1
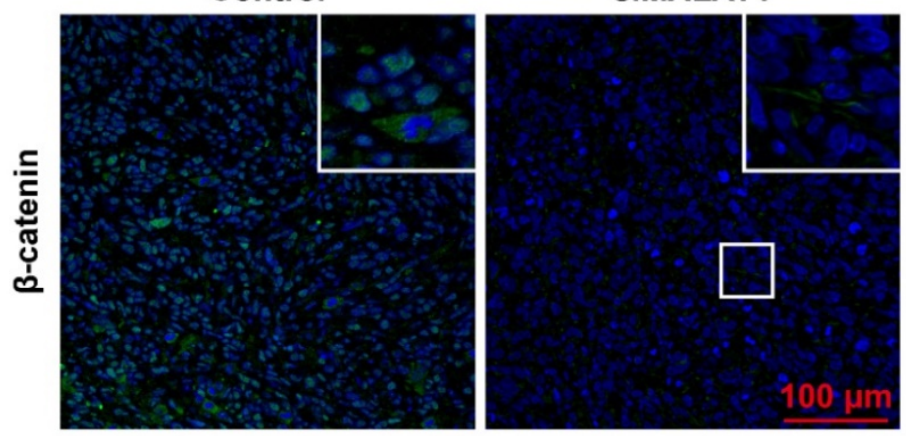

Figure 3. siMALAT1 prevents OS progression by participating in OS cell proliferation, migration and cell survival in vivo. (A) Gross photo of the tumour. Scale bar: $10 \mathrm{~mm}$. (B) In vivo proliferation rate of OS cells observed using Ki67 immunofluorescence staining. Scale bar: $100 \mu \mathrm{m}$. (C) The in vivo apoptotic rate of OS cells observed using cleaved-caspase- 3 immunofluorescence staining. Scale bar: $100 \mu \mathrm{m}$. (D) The in vivo distribution of $\beta$-catenin in cells, observed using $\beta$-catenin immunofluorescence staining. Scale bar: $100 \mu \mathrm{m}$. 
The level of spontaneous apoptosis induced by the barren microenvironment was also much higher in the siMALAT1 group than in the control group (Fig. 3C).

Even more interestingly, the distribution of $\beta$-catenin was located on the cell surface in the inactive state in the siMALAT1 group, while the $\beta$-catenin was distributed among cytomembrane, cytoplasm and nuclei in the other groups (Fig. 3D).

\section{Discussion}

There are still many unknowns regarding the principal understanding of OS biology, including indistinct aetiology, lack of biomarkers and rapid metastasizing potential. [36] Although there are several current treatment strategies, including surgical resection with pre- and post-operative chemotherapy, radiation, chemoembolization, angio-embolization, thermal therapy, radiofrequency catheter ablation and cryotherapy, the therapeutic outcome has remained disappointing. [37-39] OS presents a rigorous challenge to etiological treatment, but it is highly possible that future therapies will be established based specifically on genetic engineering, even based on the epigenetic regulation in which non-coding RNAs such as lncRNA play a role.

MALAT1, a lncRNA, has been reported several times in various types of malignant tumours. [40] However, understanding of its crucial function remains limited and more in-depth research is needed. Initially, we confirmed the high expression of MALAT1 in human OS compared to normal osteogenic tissue. However, we also confirmed that MALAT1 plays the most important role in the progression of osteosarcoma, being involved in proliferation, migration and anti-apoptosis, in line with previous reports. [21] Furthermore, we confirmed that MALAT1 could influence the expression level and re-localization of $\beta$-catenin via the MALAT1/EZH2 axis. MALAT1 can certainly increase the expression level of $\beta$-catenin, and inhibit its re-location onto the cell surface via the reduction of E-cadherin, which binds and inhibits $\beta$-catenin. [41]

Polycomb repressive complex 2 (PRC2), which contains EZH2, could perform transcriptional inhibition through methylation of lysine 27 of histone H3 (H3K27). [42] During embryonic development, EZH2 has a vitally-important role. [43] There are numerous reports of the overexpression of EZH2 in numerous solid tumours including prostate cancer, melanoma and ovarian cancer, while tumour progression can be inhibited by targeting EZH2. [44-46] In this study, we show that EZH2 is abnormally activated by the high expression of MALAT1. While directly inhibiting EZH2 suppresses the progression of OS, it can also cause haematological diseases, including myelodysplastic syndromes (MDS), myeloproliferative neoplasms (MPN), and MDS/MPN overlap disorders. Hence, it could be a more effective and safe therapeutic strategy to target MALAT1, which causes the abnormal activation of EZH2, instead of directly blocking EZH2.

The core transducer of canonical Wnt signalling is $\beta$-catenin, which associates with TCF/LEF transcription factors, or with other gene modulators related to several developmental and pathological processes. [47, 48] However, how the activation of $\beta$-catenin is regulated remains a mystery. In the classical theory of canonical Wnt signalling, when there is no Wnt ligand binding to Frizzled and its co-receptor LRP5 or LRP6, $\beta$-catenin would be phosphorylated by GSK-3 $\beta$ and degraded by proteasomes after ubiquitination. Meanwhile, in the opposing situation, in the presence of Wnt ligand, the phosphorylation of $\beta$-catenin would be inhibited and free $\beta$-catenin would accumulate and translocate into the nucleus. $[49,50]$ Moreover, it cannot be ignored that the expression of E-cadherin could inhibit the function of $\beta$-catenin through the sequestration of $\beta$-catenin, and there is even evidence indicating that only the presence of the cytoplasmic domain of E-cadherin is needed for the E-cadherin-mediated inactivation of the expression of genes downstream of $\beta$-catenin.[51] In the present study, we observed that changes in the expression of downstream genes of $\beta$-catenin are much more significant than the changes in the total expression of $\beta$-catenin. In addition, when proteins were extracted from the membrane, cytoplasm and nucleus, we found that the E-cadherin-related re-localization contributed significantly to the changes in $\beta$-catenin function.

In various types of malignant tumours, there is a negative correlation between the expression level of E-cadherin and tumour metastasis. [52] Furthermore, losing or diminishing the expression of E-cadherin usually means poor differentiation, manifesting as highly-invasive and metastatic tumours. [53] The cadherin-mediated cell-cell junction could inhibit the invasive ability of malignant tumour cells. [52, 53] It has been reported previously that the highly-invasive subtype of OS exhibits a loss of E-cadherin. [54] Additionally, in our highly-invasive OS cell line, MNNG/HOS, the levels of migration and invasion are very high due to the low expression of E-cadherin. [55] In the present study, the loss of E-cadherin could be rescued by inhibiting MALAT1. Therefore, MALAT1 is likely to be key to the treatment of highly invasive tumours, including OS.

Interestingly, we observed that the MALAT1-induced loss of E-cadherin further induced 
the uncontrolled activity of $\beta$-catenin. It has long been reported that there is an abnormal activation of canonical Wnt signalling, which is $\beta$-catenin-dependent and could lead to the promotion of proliferation and anti-apoptotic ability in human OS cells $[56,57]$. Combining this with our findings, it is very likely that the abnormal activation of $\beta$-catenin-dependent Wnt signalling can be attributed to the loss of E-cadherin caused by the MALAT1/EZH2 axis. When treated with siMALAT1 in the present study, the expression level of c-Myc, a downstream mediator of $\beta$-catenin/TCF signalling, significantly decreased along with the decrease in proliferation and anti-apoptotic ability.

Selective in vivo gene silencing technology using siRNA has been extraordinarily useful in exploring the mechanisms of a large number of human diseases.[58] In this study, we used siRNA of MALAT1 to reveal that the 'evil backstage manipulator' of highly-malignant OS is abnormal expression and localization of $\beta$-catenin caused by the MALAT1/EZH2 axis. This present study will encourage follow-up studies to further use smart nanoparticles $[59,60]$ more effectively and provide targeted delivery of siMALAT1 not only to primary foci but also to metastatic sites via systemic administration. Off-target effects are a widespread phenomenon caused by tools for the regulation of gene expression, including siRNA [61] or sgRNA (in TALEN [62] and CRISPR/Cas9[62, 63]). Ambion ${ }^{\circledR}$ Pre-designed siRNAs are pre-designed and pre-verified to have low off-target effects compared to self-designed siRNAs, and, in previous studies, they have been used as the one single siRNA for one target [64-66], although a pool of siRNA is needed when using self-designed siRNA. Moreover, we believe that therapeutic strategies targeting MALAT1 will show further improvement and maturity in the future.

The microenvironment of malignant tumours is extremely lacking in serum components, because of uncontrolled proliferation. [35] Hence, we used serum starvation to investigate the effect of this low-nutrient microenvironment on OS cells. We also found that siMALAT1 could promote cell apoptosis not only in the simulated environment in vitro but also in vivo. Thus siMALAT1 could not only inhibit the progression of OS but also cause self-destruction of the cells deep within the tumour. This would afford an opportunity for clearance of OS cells from the body's immune system, and would enhance the effectiveness of chemotherapy. When combined with nanoscale delivery systems, including liposomes [67, 68], polymeric nanoparticles [69], extracellular vesicles [32, 70,71] or other nanoparticles [72-75],
siMALAT1 will be a smarter therapeutic strategy in the future.

\section{Conclusions}

In summary, the results of the present research suggest that chemically-modified siRNA of MALAT1 can be puissant and ground-breaking in OS therapy, as any new therapeutic strategies or adjuvant therapies of existing chemotherapy will be better able to inhibit the progression of OS.

\section{Abbreviations}

OS, osteosarcoma; mRNA, messenger RNA; lncRNAs, long non-coding RNAs; MALAT1, metastasis-associated lung adenocarcinoma transcript 1; EZH2, enhancer of zeste homolog 2; siRNA, small interfering RNA; FBS, foetal bovine serum; siMALAT1, siRNA for MALAT1; siCtrl, control siRNA; RT, reverse transcription; PCR, polymerase chain reaction; PAGE, polyacrylamide gel electrophoresis; CCK-8, Cell Counting Kit-8; OD, optical density value; PI, propidium iodide; FCM, flow cytometry; CST, Cell Signalling Technology; ARRIVE, Animal Research - Reporting of In Vivo Experiments; HE, hematoxylin-eosin; ANOVA, one-way analysis of variance; BMSCs, bone marrow stem cells; PRC2, polycomb repressive complex 2; H3K27, lysine 27 of histone H3; MDS, myelodysplastic syndromes; MPN, myeloproliferative neoplasms.

\section{Acknowledgements}

This study was supported by the National Natural Science Foundation of China [Grant No. 81572239].

\section{Author Contributions}

Conception and design: Ting Yuan, Xiao-Lin Li

Development of methodology: Ting Yuan

Acquisition of data: Zhi-Chang Zhang, Chun Tang

Analysis and interpretation of data: Jing Zhang, Yang Dong

Writing of the manuscript: Ting Yuan, Zhi-Chang Zhang

Study supervision: Xiao-Lin Li

All authors reviewed the manuscript.

\section{Competing Interests}

The authors have declared that no competing interest exists.

\section{References}

1. Valery PC, Laversanne M, Bray F. Bone cancer incidence by morphological subtype: a global assessment. Cancer Causes Control. 2015; 26: 1127-39. 
2. Mirabello L, Troisi RJ, Savage SA. Osteosarcoma incidence and survival rates from 1973 to 2004: data from the Surveillance, Epidemiology, and End Results Program. Cancer. 2009; 115: 1531-43.

3. Anfinsen KP, Devesa SS, Bray F, Troisi R, Jonasdottir TJ, Bruland OS, et al. Age-period-cohort analysis of primary bone cancer incidence rates in the United States (1976-2005). Cancer Epidemiol Biomarkers Prev. 2011; 20: 1770-7.

4. Meyers PA, Heller G, Healey J, Huvos A, Lane J, Marcove R, et al. Chemotherapy for nonmetastatic osteogenic sarcoma: the Memorial Sloan-Kettering experience. J Clin Oncol. 1992; 10: 5-15.

5. Kempf-Bielack B, Bielack SS, Jurgens H, Branscheid D, Berdel WE, Exner GU, et al. Osteosarcoma relapse after combined modality therapy: an analysis of unselected patients in the Cooperative Osteosarcoma Study Group (COSS). J Clin Oncol. 2005; 23: 559-68.

6. Kaste SC, Pratt CB, Cain AM, Jones-Wallace DJ, Rao BN. Metastases detected at the time of diagnosis of primary pediatric extremity osteosarcoma at diagnosis: imaging features. Cancer. 1999; 86: 1602-8.

7. Kansara M, Teng MW, Smyth MJ, Thomas DM. Translational biology of osteosarcoma. Nat Rev Cancer. 2014; 14: 722-35.

8. Mendell JT. Targeting a Long Noncoding RNA in Breast Cancer. N Engl J Med. 2016: 374: 2287-9.

9. Iyer MK, Niknafs YS, Malik R, Singhal U, Sahu A, Hosono Y, et al. The landscape of long noncoding RNAs in the human transcriptome. Nat Genet. 2015; 47: 199-208.

10. Zhuang J, Shen L, Yang L, Huang X, Lu Q, Cui Y, et al. TGF $\beta 1$ Promotes Gemcitabine Resistance through Regulating the LncRNA-LET/NF90/miR-145 Signaling Axis in Bladder Cancer. Theranostics. 2017; 7: 3053-67.

11. Zhang Y, Cai S, Jia Y, Qi C, Sun J, Zhang H, et al. Decoding Noncoding RNAs: Role of MicroRNAs and Long Noncoding RNAs in Ocular Neovascularization. Theranostics. 2017; 7: 3155-67.

12. Zhou S, Wang J, Zhang Z. An emerging understanding of long noncoding RNAs in kidney cancer. J Cancer Res Clin Oncol. 2014; 140: 1989-95.

13. Huo $Y$, Li Q, Wang X, Jiao X, Zheng J, Li Z, et al. MALAT1 predicts poor survival in osteosarcoma patients and promotes cell metastasis through associating with EZH2. Oncotarget. 2017.

14. Ji P, Diederichs S, Wang W, Boing S, Metzger R, Schneider PM, et al. MALAT-1, a novel noncoding RNA, and thymosin beta4 predict metastasis and survival in early-stage non-small cell lung cancer. Oncogene. 2003; 22: 8031-41.

15. Gao KT, Lian D. Long non-coding RNA MALAT1 is an independent prognostic factor of osteosarcoma. Eur Rev Med Pharmacol Sci. 2016; 20: 3561-5

16. Zhang Y, Dai Q, Zeng F, Liu H. MALAT1 Promotes the Proliferation and Metastasis of Osteosarcoma Cells By Activating the Rac1/JNK Pathway Via Targeting MiR-509. Oncol Res. 2017.

17. Wang J, Sun G. FOXO1-MALAT1-miR-26a-5p Feedback Loop Mediates Proliferation and Migration in Osteosarcoma Cells. Oncol Res. 2017.

18. Liu K, Huang J, Ni J, Song D, Ding M, Wang J, et al. MALAT1 promotes osteosarcoma development by regulation of HMGB1 via miR-142-3p and miR-129-5p. Cell Cycle. 2017; 16: 578-87.

19. Li P, Zhang X, Wang H, Wang L, Liu T, Du L, et al. MALAT1 Is Associated with Poor Response to Oxaliplatin-Based Chemotherapy in Colorectal Cancer Patients and Promotes Chemoresistance through EZH2. Mol Cancer Ther. 2017; 16: 739-51.

20. Wang W, Zhu Y, Li S, Chen X, Jiang G, Shen Z, et al. Long noncoding RNA MALAT1 promotes malignant development of esophageal squamous cell carcinoma by targeting beta-catenin via Ezh2. Oncotarget. 2016; 7: 25668-82.

21. Hirata H, Hinoda Y, Shahryari V, Deng G, Nakajima K, Tabatabai ZL, et al. Long Noncoding RNA MALAT1 Promotes Aggressive Renal Cell Carcinoma through Ezh2 and Interacts with miR-205. Cancer Res. 2015; 75: 1322-31.

22. Li Y, Wu Z, Yuan J, Sun L, Lin L, Huang N, et al. Long non-coding RNA MALAT1 promotes gastric cancer tumorigenicity and metastasis by regulating vasculogenic mimicry and angiogenesis. Cancer Lett. 2017; 395: 31-44.

23. Van den Bossche J, Malissen B, Mantovani A, De Baetselier P, Van Ginderachter JA. Regulation and function of the E-cadherin/catenin complex in cells of the monocyte-macrophage lineage and DCs. Blood. 2012; 119: 1623-33

24. Nelson WJ, Nusse R. Convergence of Wnt, beta-catenin, and cadherin pathways. Science. 2004; 303: 1483-7.

25. Banan M, Puri N. The ins and outs of RNAi in mammalian cells. Curr Pharm Biotechnol. 2004; 5: 441-50.

26. Lee DH, Qi J, Bradner JE, Said JW, Doan NB, Forscher C, et al. Synergistic effect of JQ1 and rapamycin for treatment of human osteosarcoma. International journal of cancer. 2015; 136: 2055-64.

27. Hasei J, Sasaki T, Tazawa H, Osaki S, Yamakawa Y, Kunisada T, et al. Dual programmed cell death pathways induced by p53 transactivation overcome resistance to oncolytic adenovirus in human osteosarcoma cells. Mol Cancer Ther. 2013; 12: 314-25.

28. Verma SK, Tian X, LaFrance LV, Duquenne C, Suarez DP, Newlander KA, et al. Identification of Potent, Selective, Cell-Active Inhibitors of the Histone Lysine Methyltransferase EZH2. ACS medicinal chemistry letters. 2012; 3: 1091-6.

29. Amatangelo MD, Garipov A, Li H, Conejo-Garcia JR, Speicher DW, Zhang R. Three-dimensional culture sensitizes epithelial ovarian cancer cells to EZH2 methyltransferase inhibition. Cell Cycle. 2013; 12: 2113-9.
30. Guo SC, Tao SC, Yin WJ, Oi X, Yuan T, Zhang CQ. Exosomes derived from platelet-rich plasma promote the re-epithelization of chronic cutaneous wounds via activation of YAP in a diabetic rat model. Theranostics. 2017; 7: 81-96.

31. Span LF, Pennings AH, Vierwinden G, Boezeman JB, Raymakers RA, de Witte T. The dynamic process of apoptosis analyzed by flow cytometry using Annexin- $\mathrm{V} /$ propidium iodide and a modified in situ end labeling technique. Cytometry. 2002; 47: 24-31.

32. Guo SC, Tao SC, Yin WJ, Qi X, Sheng JG, Zhang CQ. Exosomes from Human Synovial-Derived Mesenchymal Stem Cells Prevent Glucocorticoid-Induced Osteonecrosis of the Femoral Head in the Rat. Int J Biol Sci. 2016; 12: 1262-72.

33. Tao SC, Yuan T, Zhang YL, Yin WJ, Guo SC, Zhang CQ. Exosomes derived from miR-140-5p-overexpressing human synovial mesenchymal stem cells enhance cartilage tissue regeneration and prevent osteoarthritis of the knee in a rat model. Theranostics. 2017; 7: 180-95.

34. Benslimane-Ahmim Z, Pereira J, Lokajczyk A, Dizier B, Galy-Fauroux I, Fischer AM, et al. Osteoprotegerin regulates cancer cell migration through SDF-1/CXCR4 axis and promotes tumour development by increasing neovascularization. Cancer Lett. 2017; 395: 11-9.

35. Jiang B, Zhang J, Xia J, Zhao W, Wu Y, Shi M, et al. IDH1 Mutation Promotes Tumorigenesis by Inhibiting JNK Activation and Apoptosis Induced by Serum Starvation. Cell Rep. 2017; 19: 389-400.

36. Luetke A, Meyers PA, Lewis I, Juergens H. Osteosarcoma treatment - where do we stand? A state of the art review. Cancer treatment reviews. 2014; 40: 523-32.

37. Chou AJ, Geller DS, Gorlick R. Therapy for osteosarcoma: where do we go from here? Pediatric Drugs. 2008; 10: 315-27.

38. Errani C, Longhi A, Rossi G, Rimondi E, Biazzo A, Toscano A, et al. Palliative therapy for osteosarcoma. Expert Review of Anticancer Therapy. 2011; 11: 217-27.

39. Dincbas FO, Koca S, Mandel NM, Hiz M, Dervisoglu S, Secmezacar H, et al. THE ROLE OF PREOPERATIVE RADIOTHERAPY IN NONMETASTATIC HIGH-GRADE OSTEOSARCOMA OF THE EXTREMITIES FOR LIMB-SPARING SURGERY. International Journal of Radiation Oncology Biology Physics. 2005; 62: 820-8.

40. Gutschner T, Hammerle M, Diederichs S. MALAT1 -- a paradigm for long noncoding RNA function in cancer. J Mol Med (Berl). 2013; 91: 791-801.

41. Lu Z, Ghosh S, Wang Z, Hunter T. Downregulation of caveolin-1 function by EGF leads to the loss of E-cadherin, increased transcriptional activity of beta-catenin, and enhanced tumor cell invasion. Cancer Cell. 2003; 4: 499-515.

42. Margueron R, Reinberg D. The Polycomb complex PRC2 and its mark in life. Nature. 2011; 469: 343-9.

43. O'Carroll D, Erhardt S, Pagani M, Barton SC, Surani MA, Jenuwein T. The polycomb-group gene Ezh2 is required for early mouse development. Mol Cell Biol. 2001: 21: 4330-6.

44. Varambally S, Dhanasekaran SM, Zhou M, Barrette TR, Kumar-Sinha C, Sanda $\mathrm{MG}$, et al. The polycomb group protein EZH2 is involved in progression of prostate cancer. Nature. 2002; 419: 624-9.

45. Zingg D, Debbache J, Schaefer SM, Tuncer E, Frommel SC, Cheng P, et al. The epigenetic modifier EZH2 controls melanoma growth and metastasis through silencing of distinct tumour suppressors. Nature communications. 2015; 6: 6051.

46. Bitler BG, Aird KM, Garipov A, Li H, Amatangelo M, Kossenkov AV, et al. Synthetic lethality by targeting EZH2 methyltransferase activity in ARID1A-mutated cancers. Nature medicine. 2015; 21: 231-8.

47. Harada N, Tamai Y, Ishikawa T, Sauer B, Takaku K, Oshima M, et al. Intestinal polyposis in mice with a dominant stable mutation of the beta-catenin gene. The EMBO journal. 1999; 18: 5931-42.

48. Nusse R. Wnt signaling. Cold Spring Harbor perspectives in biology. 2012; 4

49. Staal FJ, Luis TC, Tiemessen MM. WNT signalling in the immune system: WNT is spreading its wings. Nature reviews Immunology. 2008; 8: 581-93.

50. Pandey MK, DeGrado TR. Glycogen Synthase Kinase-3 (GSK-3)-Targeted Therapy and Imaging. Theranostics. 2016; 6: 571-93.

51. Gottardi CJ, Wong E, Gumbiner BM. E-cadherin suppresses cellular transformation by inhibiting beta-catenin signaling in an adhesion-independent manner. The Journal of cell biology. 2001; 153: 1049-60.

52. Hirohashi S. Inactivation of the E-cadherin-mediated cell adhesion system in human cancers. Am J Pathol. 1998; 153: 333-9.

53. Kawanishi J, Kato J, Sasaki K, Fujii S, Watanabe N, Niitsu Y. Loss of E-cadherin-dependent cell-cell adhesion due to mutation of the beta-catenin gene in a human cancer cell line, HSC-39. Mol Cell Biol. 1995; 15: 1175-81.

54. Kashima T, Nakamura K, Kawaguchi J, Takanashi M, Ishida T, Aburatani H, et al. Overexpression of cadherins suppresses pulmonary metastasis of osteosarcoma in vivo. International journal of cancer. 2003; 104: 147-54

55. Liu SH, Lee WJ, Lai DW, Wu SM, Liu CY, Tien HR, et al. Honokiol confers immunogenicity by dictating calreticulin exposure, activating ER stress and inhibiting epithelial-to-mesenchymal transition. Mol Oncol. 2015; 9: 834-49.

56. Chen C, Zhao M, Tian A, Zhang X, Yao Z, Ma X. Aberrant activation of Wnt/beta-catenin signaling drives proliferation of bone sarcoma cells. Oncotarget. 2015; 6: 17570-83.

57. Leal LF, Bueno AC, Gomes DC, Abduch R, de Castro M, Antonini SR. Inhibition of the Tcf/beta-catenin complex increases apoptosis and impairs adrenocortical tumor cell proliferation and adrenal steroidogenesis. Oncotarget. 2015; 6: 43016-32. 
58. Dahlman JE, Barnes C, Khan OF, Thiriot A, Jhunjunwala S, Shaw TE, et al. In vivo endothelial siRNA delivery using polymeric nanoparticles with low molecular weight. Nature nanotechnology. 2014; 9: 648-55.

59. Li Y, Liu R, Shi Y, Zhang Z, Zhang X. Zwitterionic poly(carboxybetaine)-based cationic liposomes for effective delivery of small interfering RNA therapeutics without accelerated blood clearance phenomenon. Theranostics. 2015; 5: 583-96.

60. Hong CA, Nam YS. Functional nanostructures for effective delivery of small interfering RNA therapeutics. Theranostics. 2014; 4: 1211-32.

61. Jackson AL, Linsley PS. Recognizing and avoiding siRNA off-target effects for target identification and therapeutic application. Nature reviews Drug discovery. 2010; 9: 57-67.

62. Wang X, Wang Y, Wu X, Wang J, Wang Y, Qiu Z, et al. Unbiased detection of off-target cleavage by CRISPR-Cas9 and TALENs using integrase-defective lentiviral vectors. Nature biotechnology. 2015; 33: 175-8.

63. Tsai SQ, Zheng Z, Nguyen NT, Liebers M, Topkar VV, Thapar V, et al. GUIDE-seq enables genome-wide profiling of off-target cleavage by CRISPR-Cas nucleases. Nature biotechnology. 2015; 33: 187-97.

64. Okada Y, Yamagata K, Hong K, Wakayama T, Zhang Y. A role for the elongator complex in zygotic paternal genome demethylation. Nature. 2010; 463: 554-8.

65. Vlotides G, Cooper O, Chen YH, Ren SG, Greenman Y, Melmed S. Heregulin regulates prolactinoma gene expression. Cancer Res. 2009; 69: 4209-16.

66. Siu MK, Chan HY, Kong DS, Wong ES, Wong OG, Ngan HY, et al. p21-activated kinase 4 regulates ovarian cancer cell proliferation, migration, and invasion and contributes to poor prognosis in patients. Proceedings of the National Academy of Sciences of the United States of America. 2010; 107: $18622-7$.

67. Xing H, Hwang K, Lu Y. Recent Developments of Liposomes as Nanocarriers for Theranostic Applications. Theranostics. 2016; 6: 1336-52.

68. Guo P, Yang J, Jia D, Moses MA, Auguste DT. ICAM-1-Targeted, Lcn2 siRNA-Encapsulating Liposomes are Potent Anti-angiogenic Agents for Triple Negative Breast Cancer. Theranostics. 2016; 6: 1-13.

69. Liu D, Yang F, Xiong F, Gu N. The Smart Drug Delivery System and Its Clinical Potential. Theranostics. 2016; 6: 1306-23.

70. Tao SC, Guo SC, Li M, Ke QF, Guo YP, Zhang CQ. Chitosan Wound Dressings Incorporating Exosomes Derived from MicroRNA-126-Overexpressing Synovium Mesenchymal Stem Cells Provide Sustained Release of Exosomes and Heal Full-Thickness Skin Defects in a Diabetic Rat Model. Stem Cells Transl Med. 2017; 6: 736-47.

71. Tao SC, Yuan T, Rui BY, Zhu ZZ, Guo SC, Zhang CQ. Exosomes derived from human platelet-rich plasma prevent apoptosis induced by glucocorticoid-associated endoplasmic reticulum stress in rat osteonecrosis of the femoral head via the Akt/Bad/Bcl-2 signal pathway. Theranostics. 2017; 7: 733-50.

72. Zhang J, Zhang J, Li W, Chen R, Zhang Z, Zhang W, et al. Degradable Hollow Mesoporous Silicon/Carbon Nanoparticles for Photoacoustic Imaging-Guided Highly Effective Chemo-Thermal Tumor Therapy in Vitro and in Vivo. Theranostics. 2017; 7: 3007-20.

73. Flores O, Santra S, Kaittanis C, Bassiouni R, Khaled AS, Khaled AR, et al. PSMA-Targeted Theranostic Nanocarrier for Prostate Cancer. Theranostics. 2017; 7: 2477-94.

74. Ye J, Liu E, Gong J, Wang J, Huang Y, He H, et al. High-Yield Synthesis of Monomeric LMWP(CPP)-siRNA Covalent Conjugate for Effective Cytosolic Delivery of siRNA. Theranostics. 2017; 7: 2495-508.

75. Yang T, Zhao P, Rong Z, Li B, Xue H, You J, et al. Anti-tumor Efficiency of Lipid-coated Cisplatin Nanoparticles Co-loaded with MicroRNA-375. Theranostics. 2016; 6: 142-54. 